\title{
Role of pregnancy and female sex steroids on aneurysm formation, growth, and rupture: a systematic review of the literature
}

\author{
Milli Desai, MHS, ${ }^{1}$ Arvin R. Wali, MD, MAS, ${ }^{2}$ Harjus S. Birk, MD, ${ }^{2}$ David R. Santiago-Dieppa, MD, ${ }^{2}$ \\ and Alexander A. Khalessi, MD, MS²
}

${ }^{1}$ School of Medicine and ${ }^{2}$ Department of Neurological Surgery, University of California, San Diego, California

OBJECTIVE Women have been shown to have a higher risk of cerebral aneurysm formation, growth, and rupture than men. The authors present a review of the recently published neurosurgical literature that studies the role of pregnancy and female sex steroids, to provide a conceptual framework with which to understand the various risk factors associated with cerebral aneurysms in women at different stages in their lives.

METHODS The PubMed database was searched for "“intracranial" OR "cerebral") AND "aneurysm" AND ("pregnancy" OR "estrogen" OR "progesterone")" between January 1980 and February 2019. A total of 392 articles were initially identified, and after applying inclusion and exclusion criteria, 20 papers were selected for review and analysis. These papers were then divided into two categories: 1) epidemiological studies about the formation, growth, rupture, and management of cerebral aneurysms in pregnancy; and 2) investigations on female sex steroids and cerebral aneurysms (animal studies and epidemiological studies).

RESULTS The 20 articles presented in this study include 7 epidemiological articles on pregnancy and cerebral aneurysms, 3 articles reporting case series of cerebral aneurysms treated by endovascular therapies in pregnancy, 3 epidemiological articles reporting the relationship between female sex steroids and cerebral aneurysms through retrospective case-control studies, and 7 experimental studies using animal and/or cell models to understand the relationship between female sex steroids and cerebral aneurysms. The studies in this review report similar risk of aneurysm rupture in pregnant women compared to the general population. Most ruptured aneurysms in pregnancy occur during the 3rd trimester, and most pregnant women who present with cerebral aneurysm have caesarean section deliveries. Endovascular treatment of cerebral aneurysms in pregnancy is shown to provide a new and safe form of therapy for these cases. Epidemiological studies of postmenopausal women show that estrogen hormone therapy and later age at menopause are associated with a lower risk of cerebral aneurysm than in matched controls. Experimental studies in animal models corroborate this epidemiological finding; estrogen deficiency causes endothelial dysfunction and inflammation, which may predispose to the formation and rupture of cerebral aneurysms, while exogenous estrogen treatment in this population may lower this risk.

CONCLUSIONS The aim of this work is to equip the neurosurgical and obstetrical/gynecological readership with the tools to better understand, critique, and apply findings from research on sex differences in cerebral aneurysms.

https://thejns.org/doi/abs/10.3171/2019.4.FOCUS19228

KEYWORDS cerebral aneurysm; pregnancy; female sex steroids; estrogen

$\mathrm{P}$ HYSIOLOGICAL changes that occur during a woman's lifetime may predispose her to different levels of risk for cerebral aneurysm formation, growth, and rupture. Female sex has been studied as a significant independent risk factor for intracranial aneurysm formation and growth ${ }^{10}$ and the International Study of Unruptured Intracranial Aneurysms evaluated 4060 patients and found that $75 \%$ were women. ${ }^{32}$ Two instances during which women are believed to have a unique risk for the development or rupture of cerebral aneurysms due to hormonal and hemo-

ABBREVIATIONS aSAH = aneurysmal SAH; $\mathrm{AVM}=$ arteriovenous malformation; $\mathrm{CCA}=$ common carotid artery; $\mathrm{Cl}=$ confidence interval; $\mathrm{C}$-section = caesarean section; $\mathrm{GDC}=$ Guglielmi detachable coil; HBMEC = human brain microvascular endothelial cell; HRT = hormone replacement therapy; OR = odds ratio; SAH = subarachnoid hemorrhage.

SUBMITTED March 1, 2019. ACCEPTED April 12, 2019.

INCLUDE WHEN CITING DOI: 10.3171/2019.4.FOCUS19228. 
dynamic changes are during pregnancy and menopause. For example, cardiac output increases by 30\%-50\% during pregnancy and peaks by the 3 rd trimester, ${ }^{16}$ and estrogen, which is increased in pregnancy, appears to enhance cerebral blood flow. ${ }^{19}$ In contrast to pregnancy during which female sex steroids are increased, hormones such as estrogen are decreased in peri- and postmenopause. Studies suggest that this estrogen deficiency leads to endothelial dysfunction and inflammation and explains the increased risk for aneurysmal rupture in women through menopause, but the exact mechanisms remain unspecified. ${ }^{25}$

Cerebral aneurysms in pregnancy represent a rare but important cause of subarachnoid hemorrhage (SAH), as management and treatment are complicated by considerations of both the mother and the fetus. The incidence of unruptured aneurysms in pregnancy is not well established in the literature, and the rate of ruptured aneurysms ranges from 3 to 11 per 100,000 pregnancies. ${ }^{12}$ Fifty percent of all aneurysm ruptures in women younger than 40 years are reported to be pregnancy related. ${ }^{2}$ Aneurysm rupture has been shown to have greater mortality risk in pregnant patients than in nonpregnant patients. ${ }^{18}$ There are limited studies on the relationship of pregnancy and cerebral aneurysms: the incidence is rare, and additionally it is not feasible to study cohorts of pregnant women through randomized controlled trials. The risk of aneurysm rupture during pregnancy and subsequent options for treatment and delivery are controversial. Previous studies have reported an increased risk of aneurysm rupture and subsequent $\mathrm{SAH}$ during pregnancy and delivery, with as many as $77 \%$ of 154 cases of verified intracranial hemorrhage during pregnancy caused by aneurysm rupture. ${ }^{5}$ However, the aforementioned study did not include a control group and is therefore limited in interpretation, and more recent studies indicate the risk of aneurysm rupture during pregnancy is similar to that of the general population. ${ }^{11}$ Treatment modalities for aneurysms have changed since early case reports in 1965 showed the options for a ruptured aneurysm in pregnancy were intracranial surgery versus bed rest with imminent death. ${ }^{22}$ Current treatment modalities include endovascular treatment with a focus on minimizing morbidity from intracranial surgery, as well as minimizing fetal exposure to angiography. ${ }^{17}$ We rely on epidemiological data and reviews of cases to identify which trimester of pregnancy patients present with aneurysm rupture, how aneurysms grow during pregnancy, treatment options, and whether vaginal versus caesarean section (C-section) deliveries are indicated to minimize the risk of aneurysmal $\mathrm{SAH}(\mathrm{aSAH})$ during pregnancy.

Women are at highest risk for aneurysm rupture in the perimenopausal and postmenopausal state. ${ }^{4}$ The relationship between postmenopausal estrogen deficiency and aSAH has been studied and described more extensively through experimental studies using animal models. ${ }^{25}$ Understanding the current literature about experimental models to explain the pathophysiology of cerebral aneurysms in estrogen-deficient states and epidemiological data to understand population health components will shed light on the overall role of female sex steroids and hormones on the development of cerebral aneurysms. These findings can be applied to multiple scenarios of aneurysm formation, growth, and rupture. Furthermore, these findings point to the development of hormone therapy and targeted therapy to prevent the formation of aneurysms, halt growth, and prevent aneurysm rupture. Understanding the role of female sex steroids such as estrogen will also contribute to an understanding of the underlying sex disparities that exist in presentation of cerebral aneurysms. Here, we review the literature on the role of pregnancy and sex steroids on cerebral aneurysms and summarize salient findings from epidemiological and experimental studies.

\section{Methods}

A systematic review to analyze the role of pregnancy and sex steroids in cerebral aneurysms was performed through the PubMed registry with articles dating from January 1, 1980, to February 1, 2019. Search terms were "("intracranial" OR "cerebral") AND "aneurysm" AND ("pregnancy" OR "estrogen" OR "progesterone")." This search yielded a total of 392 results from the PubMed database. Articles were included within this review if they presented primary human or animal data or investigated the epidemiology of pregnancy, sex steroids (i.e., estrogen and/or progesterone), and cerebral aneurysms. Case-series analyses were included. Articles were excluded if aneurysms were not differentiated from other intracranial vascular abnormalities (i.e., arteriovenous malformations [AVMs]). Single case reports were excluded from our review because multiple articles have already synthesized the published single case reports as case series.

The extracted articles were then divided into two categories: 1) epidemiological studies about the formation, growth, rupture, and management of cerebral aneurysms in pregnancy; and 2) investigations on female sex steroids and cerebral aneurysms (animal studies and epidemiological studies). Epidemiological studies were reviewed for study design, sample size of population studied, age and gestational age if applicable, and outcomes of cerebral aneurysm formation, growth, rupture, treatment, and management. Animal studies were evaluated for study design (in vivo vs in vitro), subject type, injury model, number of animals included in experimental and control groups, and outcomes of cerebral aneurysm formation, growth, rupture, treatment, and management.

\section{Results}

Three hundred ninety-two articles were initially identified from the PubMed database. After applying inclusion and exclusion criteria, 20 papers were selected for review and analysis. Using the search criteria, no prospective or randomized controlled trials in pregnancy were identified. The 20 articles presented here include 7 epidemiological articles on pregnancy and cerebral aneurysms, 3 articles reporting case series of cerebral aneurysms treated by endovascular therapies in pregnancy, 3 epidemiological articles reporting the relationship between female sex steroids and cerebral aneurysms through retrospective case-control studies, and 7 experimental studies using animal and/or cell models to understand the relationship between female sex steroids and cerebral aneurysms. Figure 1 is a flowchart describing the paper selection process. 


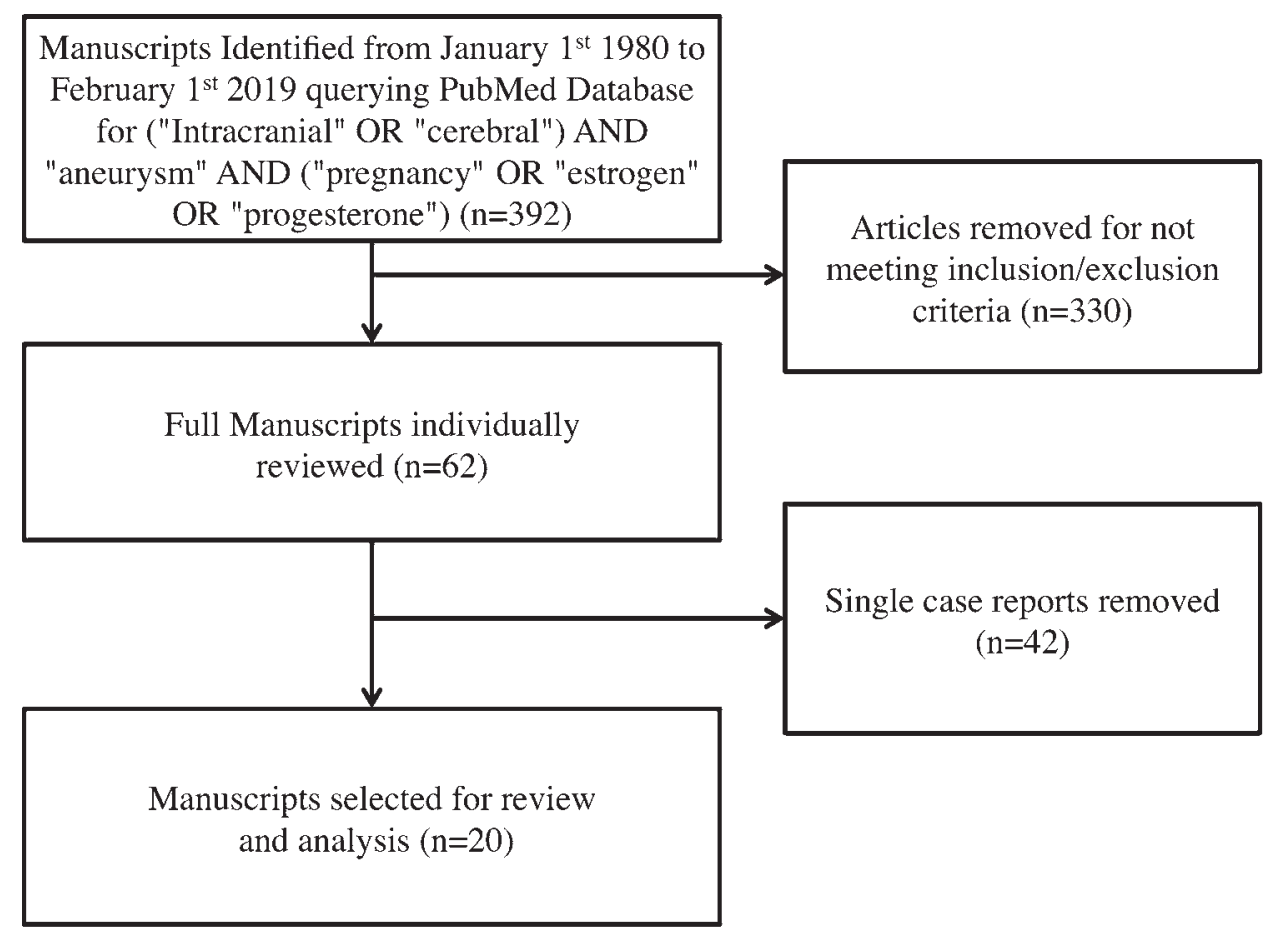

FIG. 1. Flow diagram demonstrating the search algorithm to identify literature from the PubMed database. A total of 392 articles were initially identified using our search criteria, of which 20 met the inclusion and exclusion criteria and were included within this review.

\section{Epidemiological Studies of Cerebral Aneurysms in Pregnancy}

Seven epidemiological articles reporting cerebral aneurysms in pregnancy were included. A full description of results from these investigations can be found in Table 1. Within the 7 articles, we include 1 case crossover study, 1 retrospective cohort study, 2 retrospective reviews, 1 retrospective review and literature review of cases, 1 caseseries report, and 1 literature review of cases. Among 89 cases from single case reports that have been summarized in 2 studies, 72 (approximately 80\%) represented cases of ruptured aneurysms in the 3rd trimester of pregnancy.,23 This finding is consistent with previous studies and caseseries reports that have found that aneurysms are most likely to rupture in the 3rd trimester of pregnancy and up to 6 weeks postpartum. ${ }^{12,24}$ Studies that summarized case reports also indicated aneurysm occlusion through surgical clipping in $53.8 \%$ of cases and with an endovascular procedure in $36.5 \%$ of cases, ${ }^{23}$ and coil embolization being associated with a lower complication rate than clipping in patients with ruptured aneurysms $(9.5 \%$ vs $23.1 \%) .{ }^{1}$ One study in our analysis reported that $19.8 \%$ (22 of 111 cases) of hemorrhagic stroke in pregnancy was due to aneurysm rupture..$^{33}$ In 2 longitudinal studies in our analysis with a combined sample size of 1130 patients, the relative risk of rupture during pregnancy and deliveries was comparable to the annual rupture risk in the general population $(1.4 \%, 95 \%$ confidence interval $[\mathrm{CI}] 1.35 \%-1.57 \%)$ during pregnancy and $0.05 \%$ (95\% CI $0.0468 \%-0.0634 \%$ ) during delivery; and in the second study, $0.4 \%$ (95\% CI $0.2 \%-$ $0.9 \%$ ) during pregnancy, delivery, or the puerperium. ${ }^{11,30}$ In a study of 5 aneurysms in pregnancy with monitoring of aneurysm growth, the aneurysms in 4 pregnancies did not change in size, remaining 2-5 $\mathrm{mm}$, while in 1 pregnancy, the aneurysm increased from 6 to $7 \mathrm{~mm}$ during the 3rd trimester, but returned to its original size in the postpartum period. ${ }^{28}$ Of the 5 studies we include that compared $\mathrm{C}$-section and vaginal delivery rates in pregnant women with cerebral aneurysms, all reported increased rates of Csections as the method of delivery, regardless of aneurysm rupture or nonrupture. ${ }^{1,11,23,24,28} \mathrm{~A}$ C-section followed by aneurysm treatment was reported in 1 study as the accepted delivery method for ruptured aneurysms, ${ }^{24}$ whereas in 2 other studies the widespread use of C-sections in unruptured aneurysms was reported to be unnecessary. ${ }^{11,30}$

\section{Endovascular Treatment of Cerebral Aneurysms in Pregnancy}

Three articles reporting case series of cerebral aneurysms treated by endovascular therapies in pregnancy were identified. A full description of results from these investigations can be found in Table 2. Summaries of case reports that we have included report that in 89 cases of cerebral aneurysms in pregnancy, occlusion of the aneurysm was achieved through surgical clip placement in $53.8 \%$ of cases. ${ }^{23}$ Surgical clipping of aneurysms is still used and reported in the literature, but the most recent neurosurgical literature has discussed endovascular coil embolization of aneurysms as a treatment option. In the combined 8 cases of aneurysms treated endovascularly in pregnancy that we include in this review, all were treated successfully with Guglielmi detachable coils (GDCs) and without the need 


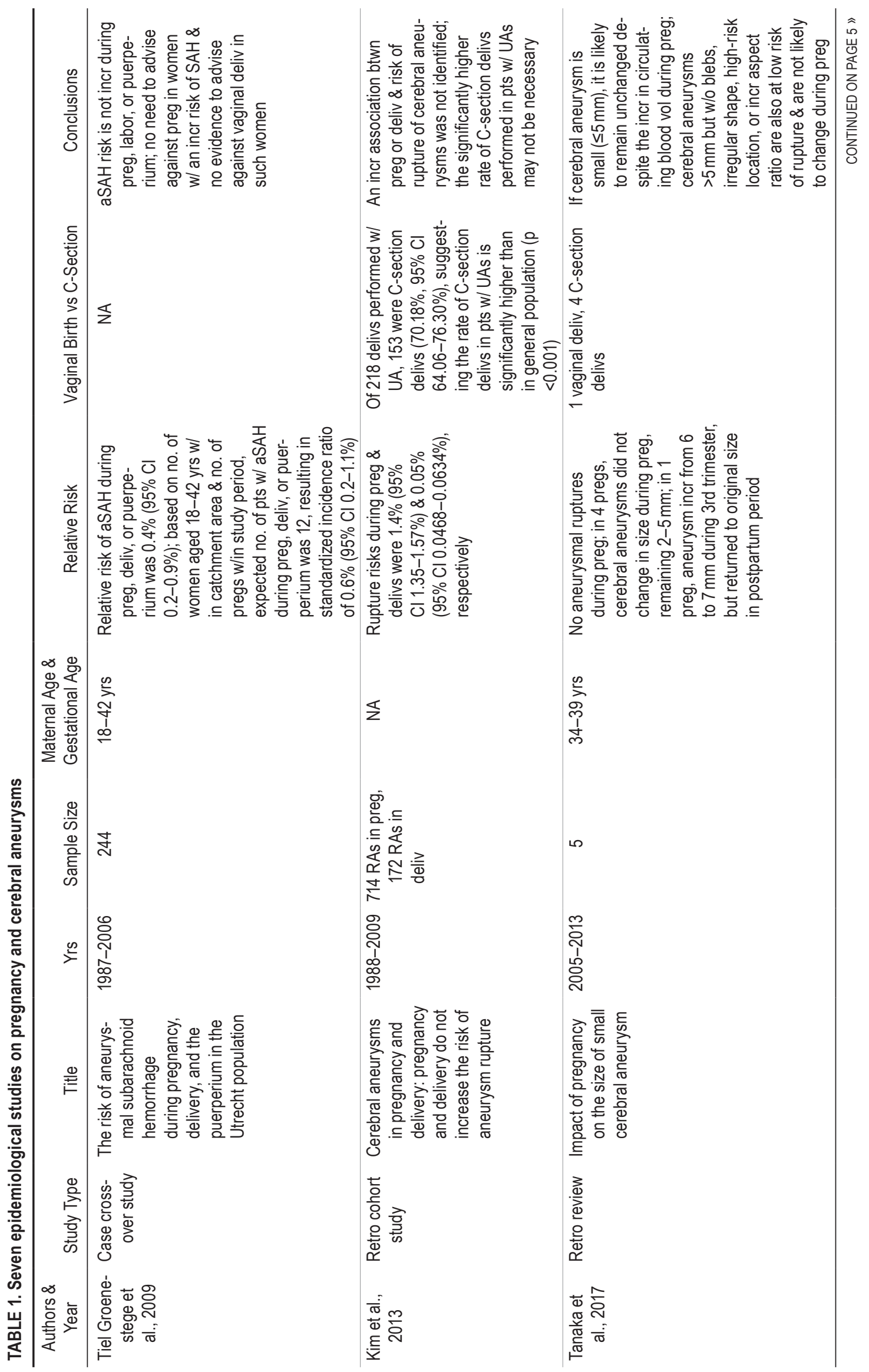


Desai et al.

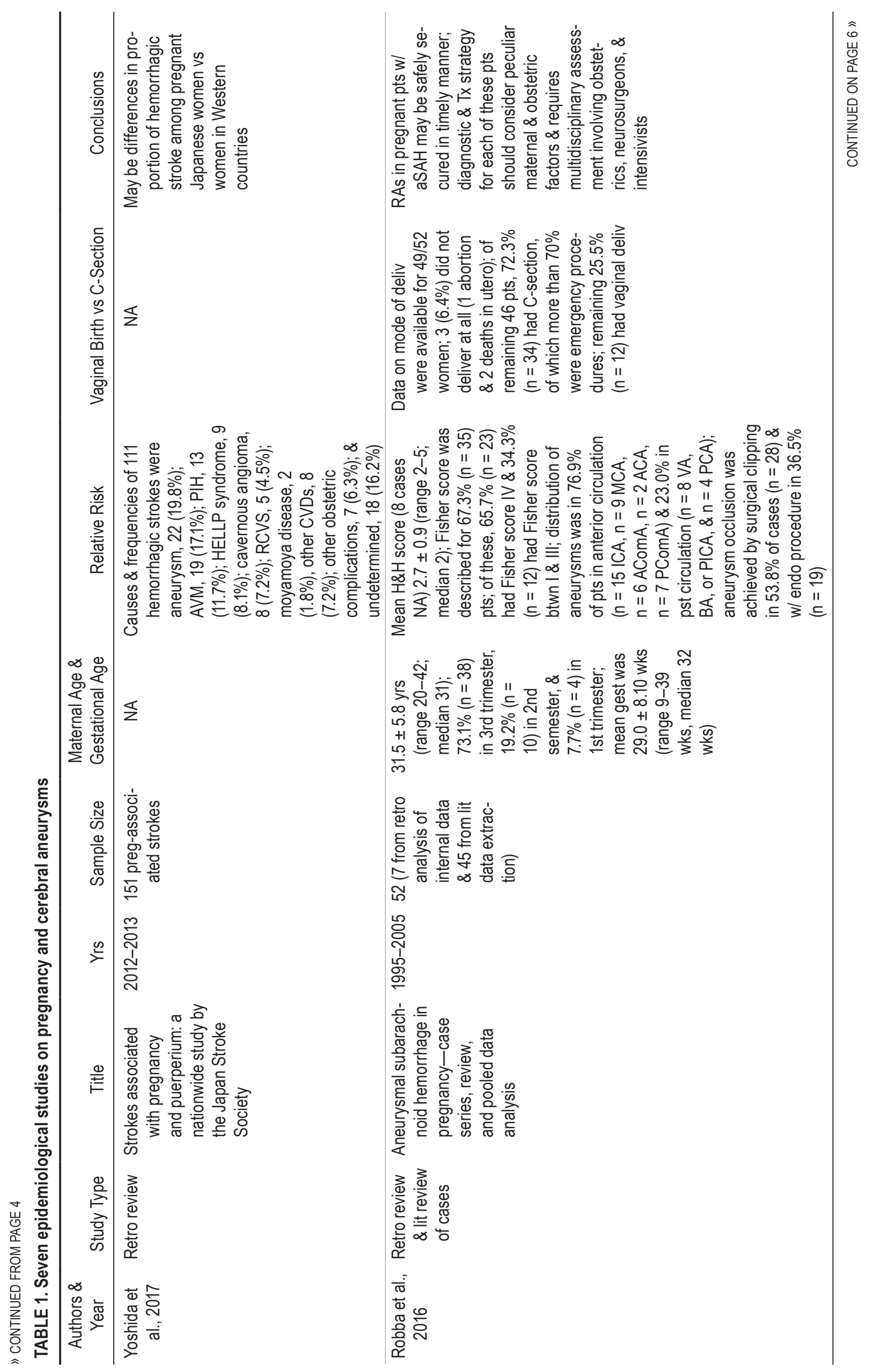




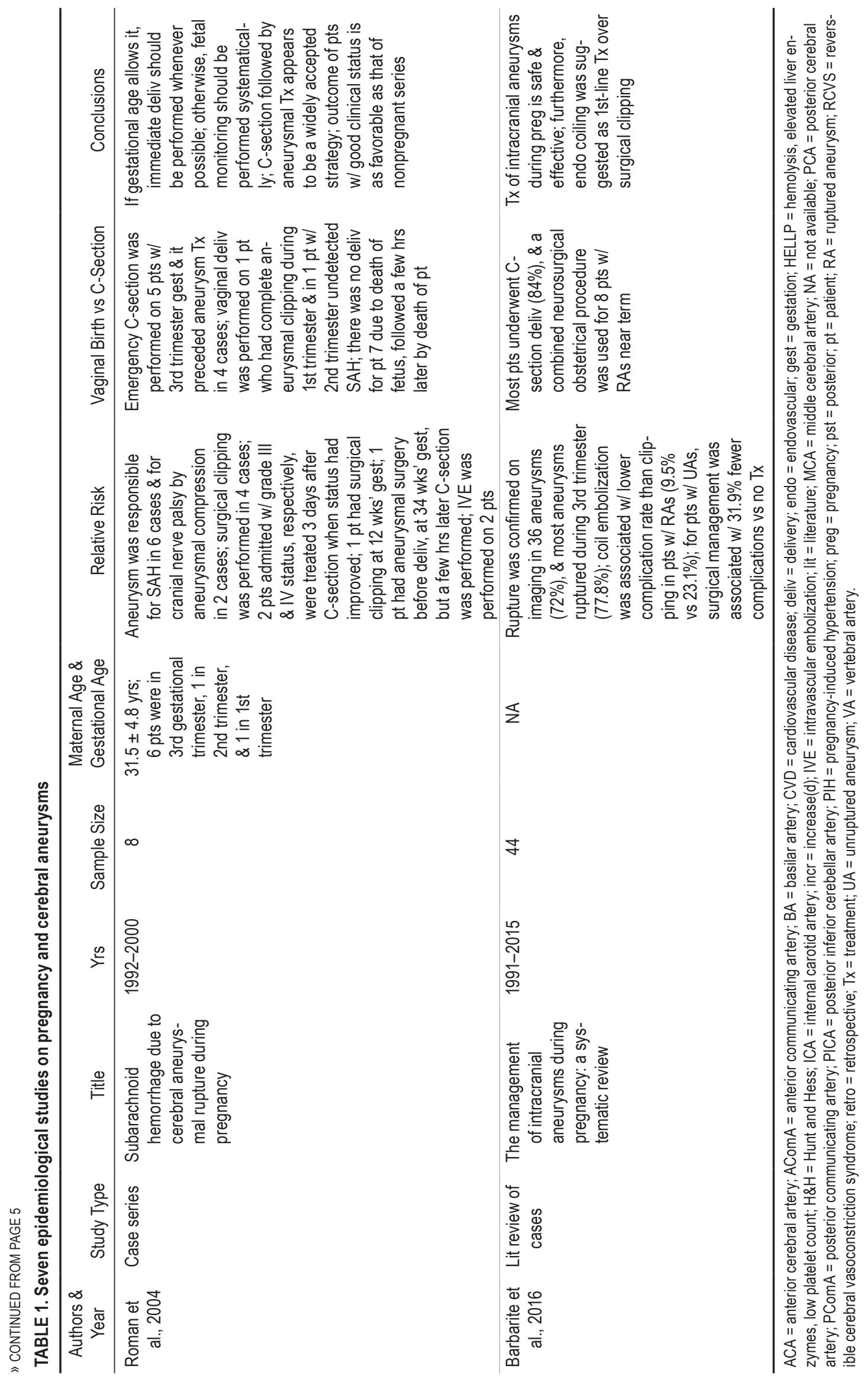




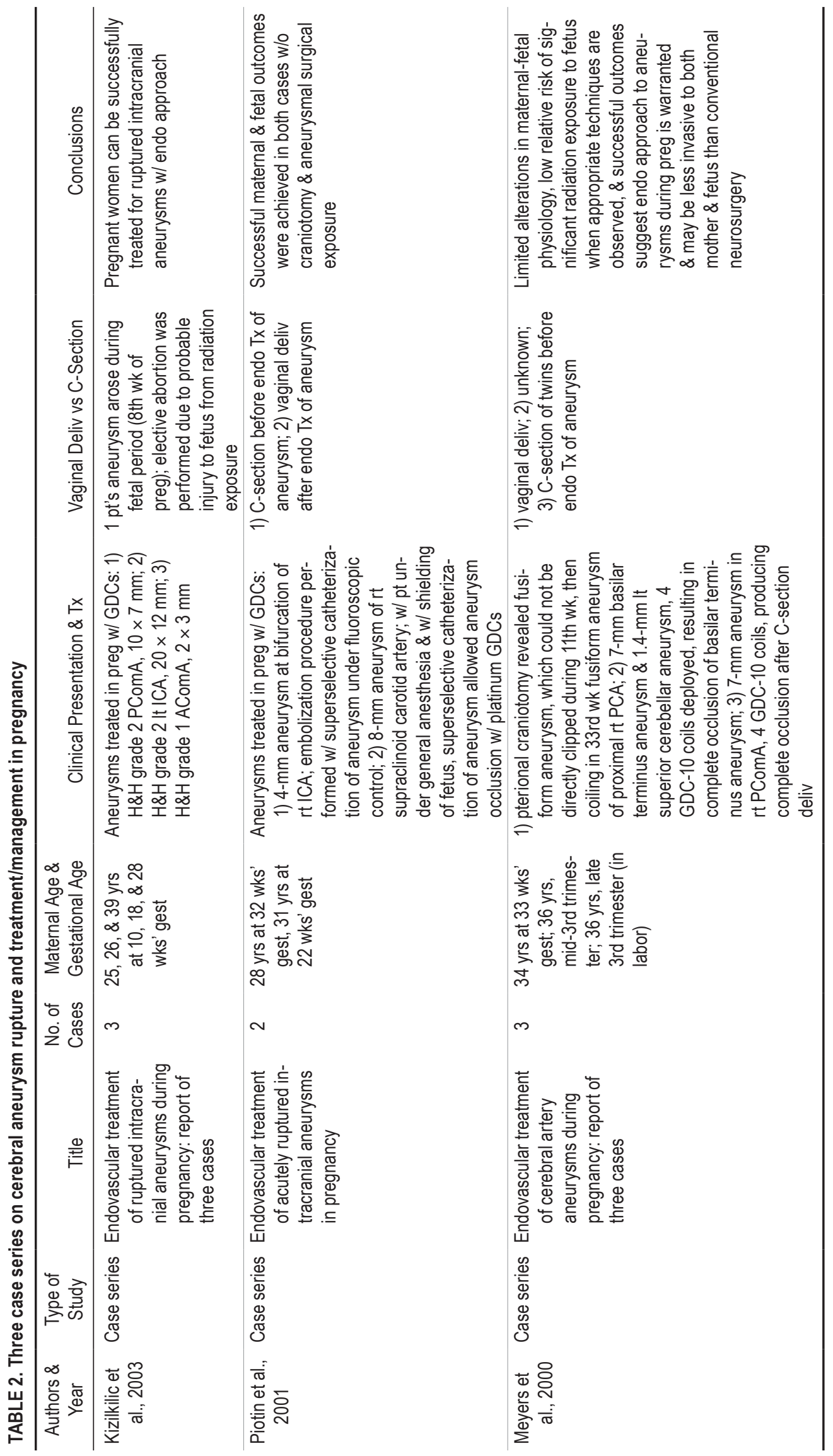


for open craniotomy. ${ }^{13,17,21}$ Endovascular treatment occurred before vaginal deliveries, as well as after C-section deliveries. ${ }^{13,17,21}$ Time of endovascular treatment ranged from 1st, 2nd, and 3rd trimester of gestation; the only adverse pregnancy outcome reported was an elective abortion after successful endovascular treatment of an aneurysm during the 8th week of gestation due to concern for fetal injury from radiation exposure. ${ }^{13}$

\section{Epidemiological Studies of Female Sex Steroids and Cerebral Aneurysms}

Three epidemiological articles reporting the relationship between female sex steroids and cerebral aneurysms through retrospective case-control studies were included. A full description of results from these investigations can be found in Table 3 . In 76 postmenopausal women with cerebral aneurysms compared to matched controls, both later menopause age (odds ratio [OR] 0.79, 95\% CI 0.63-0.996, $\mathrm{p}=0.046)$ and ever use of hormone replacement therapy (HRT; OR $0.23,95 \%$ CI $0.13-0.42$, p < 0.0001 ) were significantly associated with a lower risk of aneurysm in women in the case group. ${ }^{6}$ Conversely, and showing the same results, in 60 postmenopausal women with intradural aneurysms compared to matched controls, there was a significant association between a lower rate of oral contraceptive (OR 2.1, 95\% CI 1.17-3.81, $\mathrm{p}=0.01)$ and HRT (OR $3.09,95 \%$ CI 1.54-6.22, $\mathrm{p}=0.002$ ) use and the presence of a cerebral aneurysm. ${ }^{3}$ In a study of 233 women in which 43 had hysterectomies, the women with a history of hysterectomy had fewer large aneurysms (8\% vs $24 \%, \mathrm{p}=0.03)$, and fewer presented with a ruptured aneurysm (28\%) than the nonhysterectomy group $(51 \%, \mathrm{p}=0.004) .{ }^{20}$

\section{Experimental (animal and cell model) Studies Investigating Female Sex Steroids and Cerebral Aneurysms}

Seven experimental studies using animal and/or cell models to understand the relationship between female sex steroids and cerebral aneurysms were included. A full description of results from these experimental investigations can be found in Table 4. All 7 studies used in vivo experiments, with 1 study using both in vivo and in vitro (human brain microvascular endothelial cell [HBMEC]) experiments. Four studies used rat models, 2 studies used mice models, and 1 study used a rabbit model, for a total of 264 animals in the experimental groups, and a total of 98 animals in the control groups. Mechanisms to create cerebral aneurysms in animal models were ligation of a common carotid artery (CCA) and bilateral posterior renal arteries, ${ }^{7-9,27}$ hypertensive diet, and angiotensin $\mathrm{II}^{7}$ and injection of elastase into the CSF with deoxycorticosterone acetate salt hypertension. ${ }^{26}$ Estrogen deficiency was induced through bilateral oophorectomy ${ }^{7-9,15,26,27}$ and estrogen E2 receptor blockade. ${ }^{7}$ Ovariectomized female mice had a significantly higher incidence of aneurysms than male mice with sham ovariectomy, ${ }^{26}$ and 3 times higher incidence of cerebral aneurysm formation than females without bilateral oophorectomy. ${ }^{8}$ Estrogen deficiency has been shown to cause endothelial dysfunction, which may lead to changes in vascular wall integrity and contribute to aneurysm formation. Aneurysm changes in animal models with oopho- rectomy and HRT were limited to stage I or II, whereas most changes in animal models with oophorectomy but no HRT were identified as saccular dilation (stage III). ${ }^{9}$ Estrogen deficiency induced endothelial dysfunction and reactive oxygen species generation in animal models and HBMECs, which triggered endothelial damage that led to cerebral aneurysms. ${ }^{27}$ Estrogen deficiency may lead to inflammatory changes that contribute to aneurysm rupture. Estrogen-deficient mice had more aneurysm ruptures than control mice, and were found to upregulate IL-17A, which downregulates E-cadherin, encouraging macrophage infiltration in the aneurysm vessel wall. Hypertension is an additional risk factor for aneurysm development in animal models with estrogen deficiency.9,31 Animal models with estrogen deficiency and induced hypertension had significantly higher vascular damage scores in multiple regions of the circle of Willis, signifying that hypertension and estrogen deficiency make the circle of Willis more vulnerable to flow-induced aneurysmal remodeling and tortuosity. ${ }^{31}$ While estrogen deficiency itself has been reported by the previously mentioned studies to increase risk for cerebral aneurysm complications, exogenous estrogen treatment in an estrogen-deficient state has been shown to be protective from cerebral aneurysm complications in the following 3 studies. Treatment with estradiol, ${ }^{7}$ a selective estrogen receptor modulator such as bazedoxifene, ${ }^{15}$ and estrogen ${ }^{26}$ was shown to decrease the amount of aneurysm ruptures in animal models of estrogen deficiency.

\section{Discussion}

Sex differences in the care of patients with cerebral aneurysms provide a unique opportunity for collaboration among multiple physician specialties: neurosurgeons, obstetricians/gynecologists, perinatologists, anesthesiologists, radiologists, intensivists, and primary care providers. This systematic review describes the spectrum of risk for women in pregnancy and in estrogen-deficient states (i.e., menopause, surgical oophorectomy, etc.) and provides readers with the information that female sex steroids may impact women and their cerebrovascular anatomy differently at different stages of their life.

Studies included in this review suggest that the rate of aneurysm rupture in pregnancy is not increased compared to the general population. Neurosurgeons can be prepared to manage pregnant women with aneurysms similarly to the general population. As our review indicated, it may not be necessary to advise pregnant women with cerebral aneurysms against vaginal deliveries, and obstetricians can use these data to collaborate with neurosurgeon colleagues and provide risk stratification to their patients accordingly. ${ }^{11,30}$ An older study from 1990 reports a fetal case fatality rate of approximately $17 \%$ as a result of ruptured intracranial aneurysms in pregnancy. ${ }^{5} \mathrm{~A}$ more recent retrospective review of case reports describes a fetal case fatality rate of approximately $6.5 \% .^{23}$ In reviewing published papers, studies have not commented extensively on adverse pregnancy outcomes through the natural history of a patient with a ruptured aneurysm or through treatment, perhaps because study design is limited to retrospective and case-series formats. We hypothesize that fetal case fatality rates have de- 

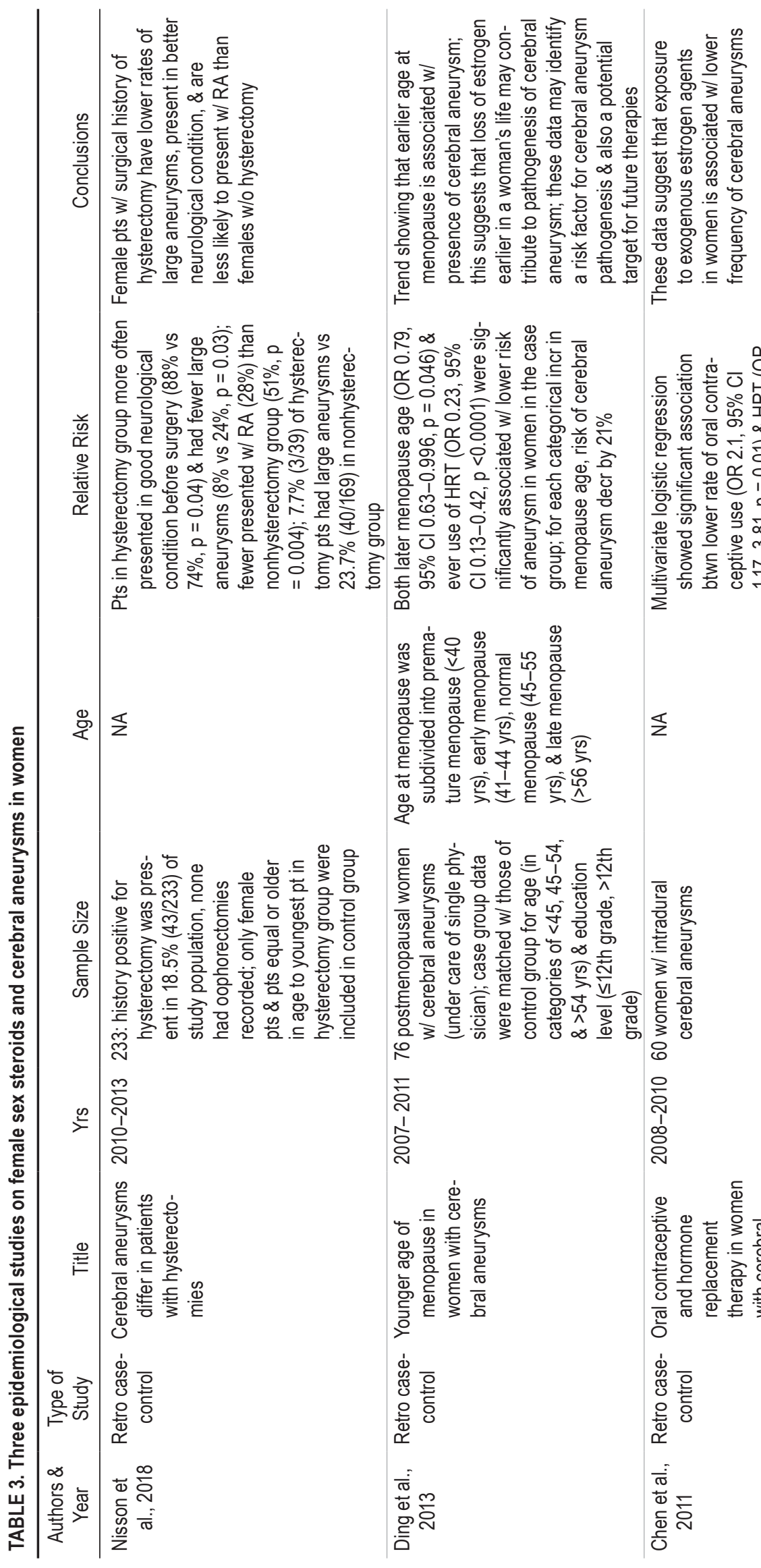

Tळळ

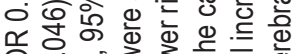

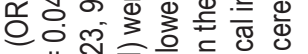

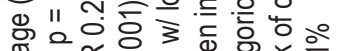
刃 ભ

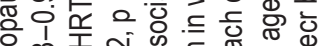

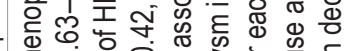

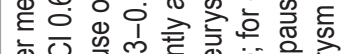

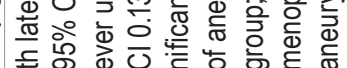
드

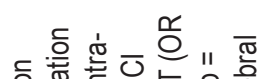

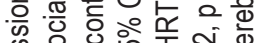
乐 ప্రু 离

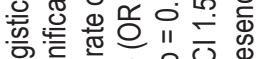

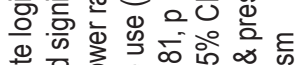
离

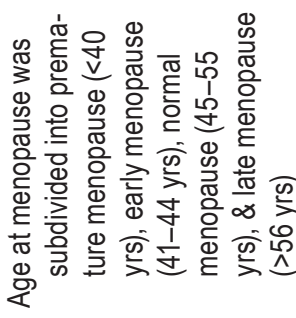

ฮ

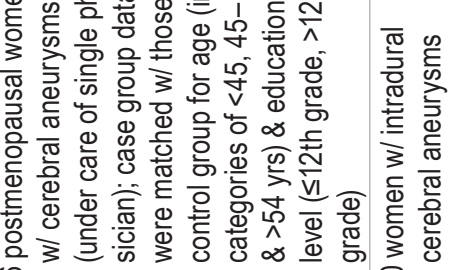
e

둥

용

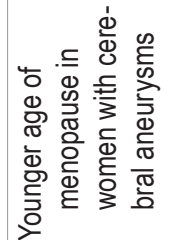

di

은 등

产

离

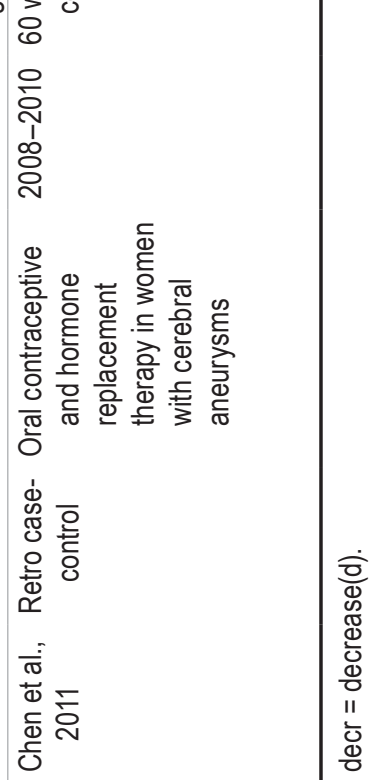




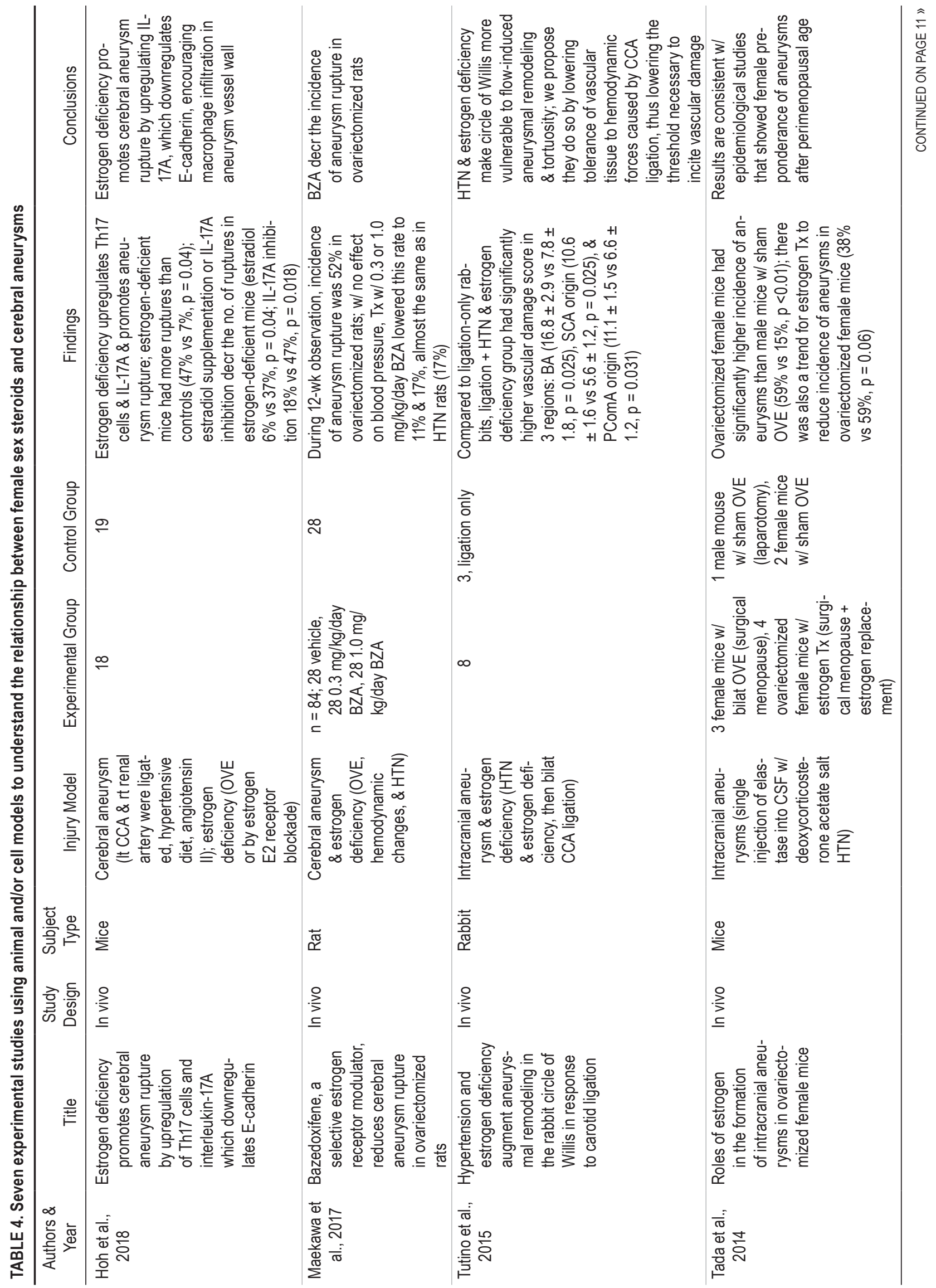




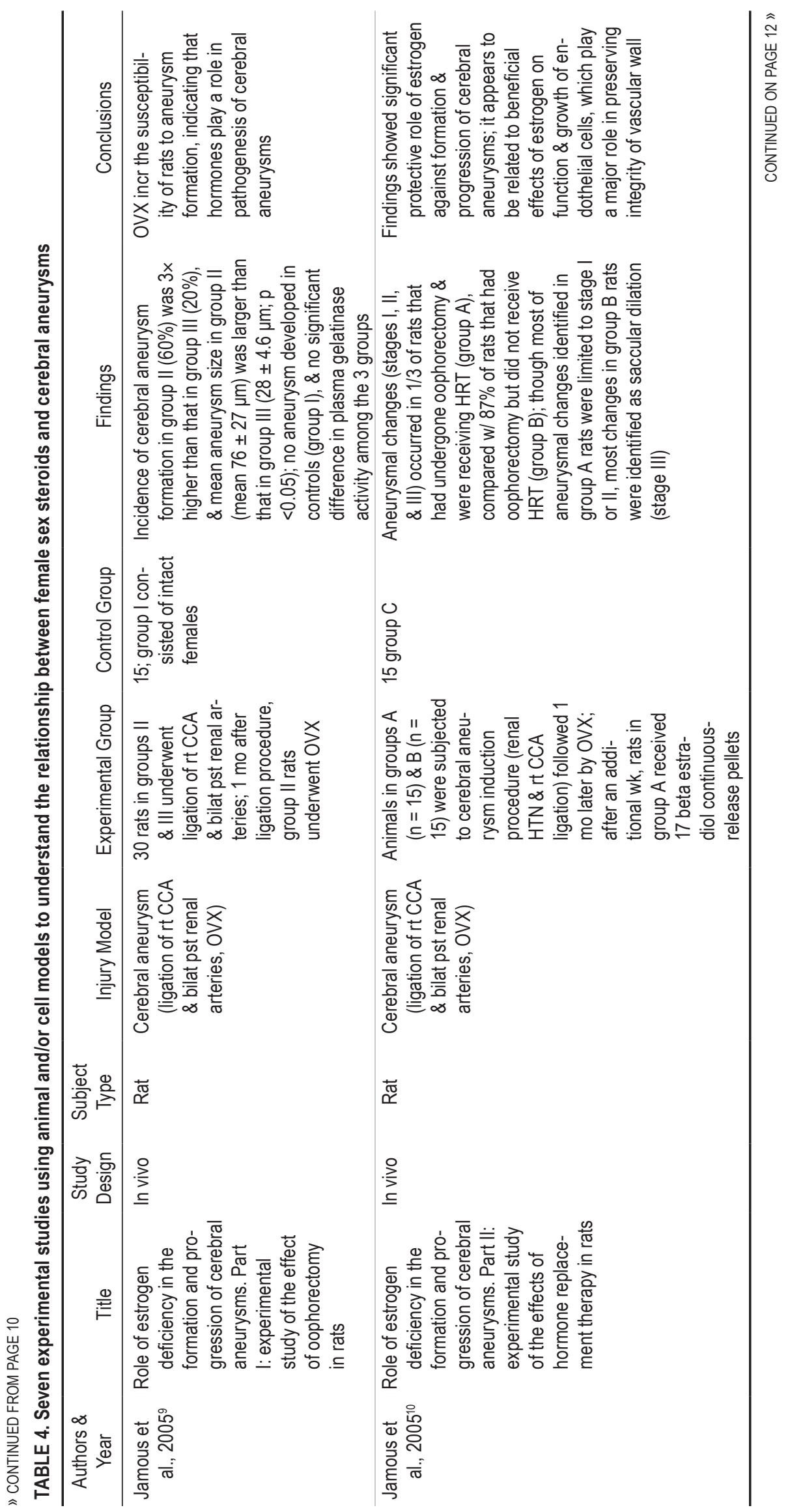




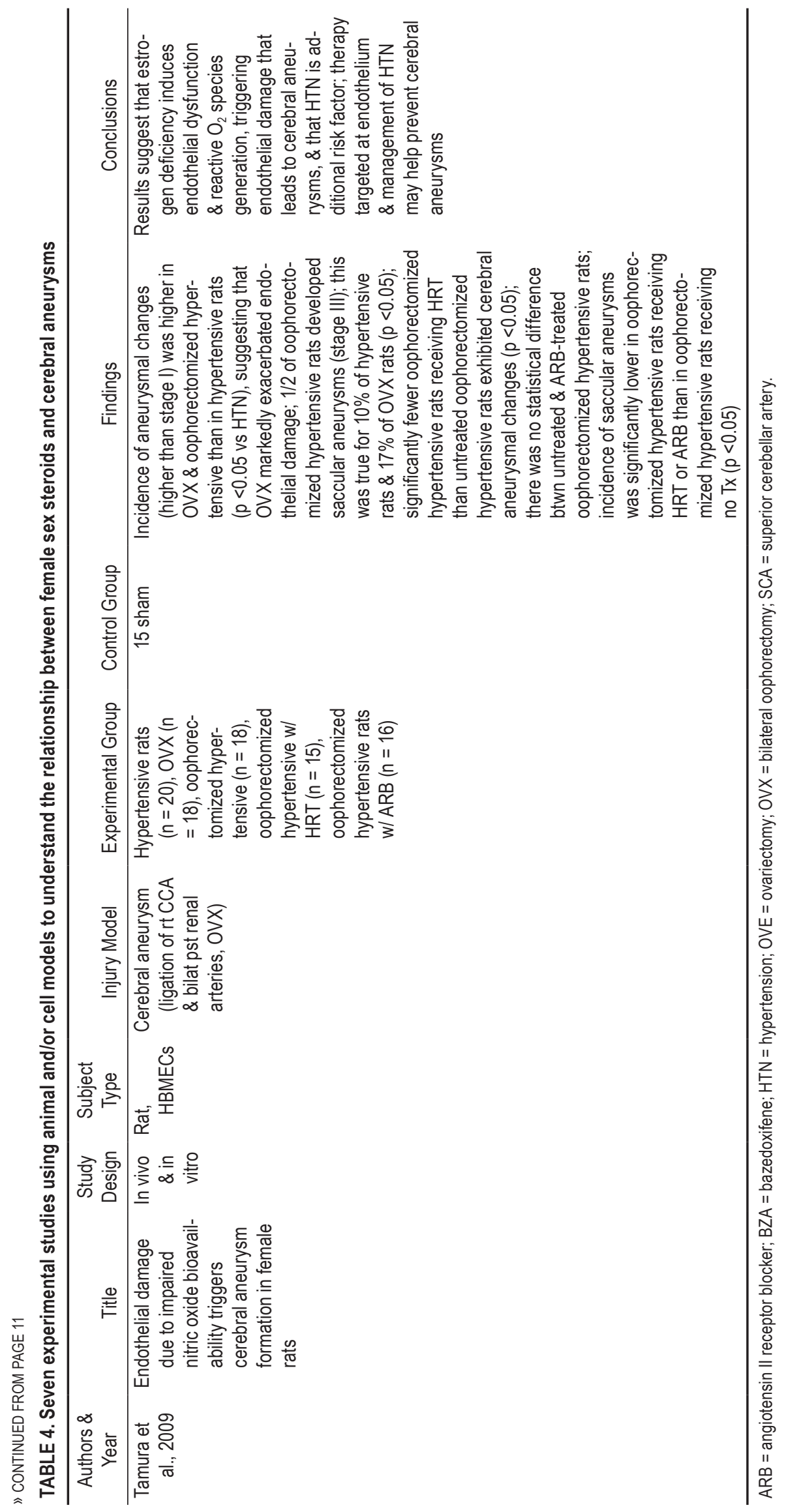




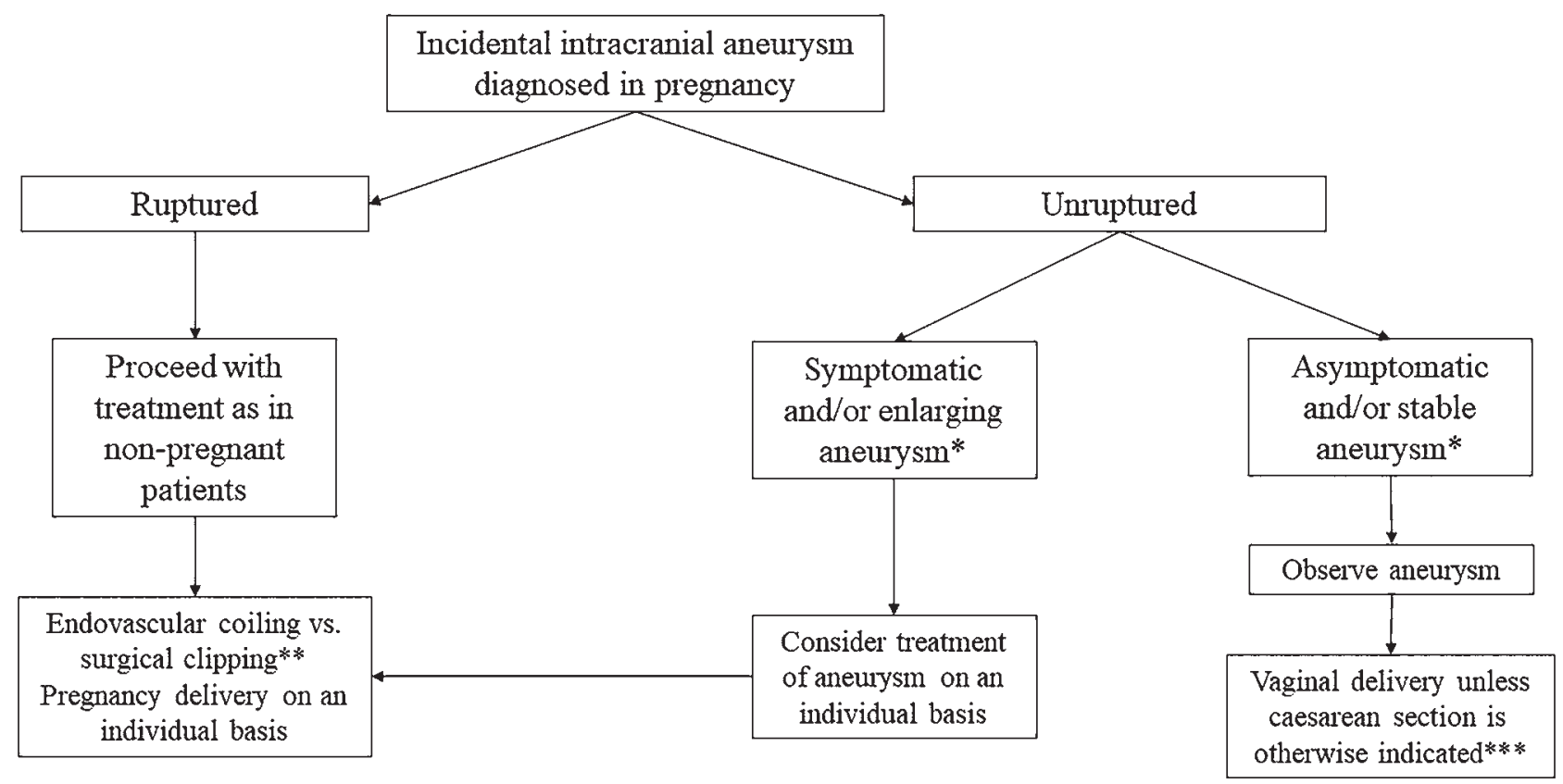

FIG. 2. Diagrammatic algorithm of how to clinically approach a patient who presents with an incidental aneurysm diagnosed during pregnancy. ${ }^{*}$ Can use MRI to evaluate aneurysm growth during pregnancy. Further research is necessary to elucidate size cutoffs that are clinically meaningful in this population. ${ }^{* *}$ Coiling thought to be a safe treatment and may be preferred to clipping in pregnancy. Consider abdominal lead shielding and radiation exposure. ${ }^{* * *}$ There is no evidence to suggest that maternal or fetal outcome is improved by C-section delivery in comparison with closely supervised vaginal delivery.

creased due to advancements in obstetric care, but further research is needed to evaluate this topic.

Based on the data that we present, we have created a diagrammatic algorithm of how to approach a patient who presents with an incidental aneurysm diagnosed during pregnancy (Fig. 2). We conclude that if unruptured intracranial aneurysms in pregnancy are stable and asymptomatic, they may be observed. Symptomatic and/or enlarging unruptured aneurysms may be treated on an individual basis. Ruptured intracranial aneurysms in pregnancy are treated as they would be in nonpregnant patients. Neurosurgical considerations generally take precedence over obstetric considerations for ruptured, symptomatic, and/ or growing intracranial aneurysms. Surgical clipping has historically been accepted as the treatment for ruptured intracranial aneurysms during pregnancy, but endovascular coiling is now believed to be a safe treatment and may be preferred to clipping. Because there are no prospective or randomized controlled trials investigating this subject, we rely on retrospective and case report data to inform our clinical decision-making. We have included 2 studies that address this topic. In a retrospective cohort analysis, endovascular coiling had lower mortality rates in pregnant women with ruptured aneurysms compared to clipping. ${ }^{11}$ In a previous literature review, endovascular coiling was found to have lower complication rates than surgical clipping in pregnant women with ruptured aneurysms. ${ }^{1}$ In both studies, surgical management of ruptured intracranial aneurysms during pregnancy was found to be superior to no treatment. Considerations for endovascular coiling of intracranial aneurysms during pregnancy include concern for harmful effects of radiation to the fetus and potential harmful effects of anticoagulation. Given that the probability of radiation damage increases with increasing absorbed dose, the radiation dose and stage of fetal development at the time of exposure should be evaluated for endovascular coiling procedures during pregnancy. An International Commission on Radiation Protection report recommended that with optimized abdominal lead shielding, coiling-related fetal radiation can be neglected. ${ }^{29}$ In the studies we include that used coiling for ruptured aneurysms, radiation exposure was limited through abdominal lead shielding, limited fluoroscopy in proximity to the uterus, and precautions to limit radiation exposure to the patient as well as adequate fetal monitoring. ${ }^{11,14,17}$ Regarding anticoagulation during the endovascular procedure, heparin is not teratogenic and has previously been used for coiling of ruptured intracranial aneurysms during pregnancy, but would need to be discontinued before a C-section. ${ }^{14}$ Further research is needed to evaluate additional endovascular treatment options such as stent-assisted coiling during pregnancy, and whether aspirin and/or clopidogrel are safe to use in this setting.

This review elucidates the potential with which neurosurgeons and gynecologists can determine how estrogen deficiency contributes to endothelial dysfunction and inflammation, which may lead to cerebral aneurysm formation, growth, and rupture as reported by multiple studies in this review. Estrogen HRT is commonly used among postmenopausal women, and this review presents animal data that suggest improvement in aneurysmal outcomes with HRT ${ }^{3,6}$ Hormonal therapies and the effect of targeted drugs on cerebral aneurysms should be further studied in human clinical trials. 
Our review suggests that estrogen deficiency in animal models contributes to aneurysm formation and rupture. $7,8,26,27,31$ Pregnancy is a high-estrogen state, which might suggest less aneurysm formation and rupture during this time through the findings of estrogen as a protective cerebrovascular factor in animal studies. However, our review of epidemiological data in pregnant women with cerebral aneurysms shows a similar rate of rupture as the general population. In addition to hormonal and hemodynamic factors, there may be additional factors inherent to pregnancy that raise the risk of aneurysm rupture compared to that of the general population, despite a high estrogen state during pregnancy. We hope this review article encourages additional longitudinal research in prospective cohorts to further characterize the underlying causes of pregnancy and sex steroid effects on cerebral aneurysms.

\section{Conclusions}

The mechanisms of cerebral aneurysm formation, growth, and rupture during pregnancy and in estrogen-deficient states are complex. This review article summarizes the current literature of hormonal- and pregnancy-related risks for cerebral aneurysms, and can help guide clinical decision-making for both neurosurgeons and obstetricians and multiple members of the healthcare team about treatment and management options for pregnant women with cerebral aneurysms. This article helps readers understand the current research on estrogen deficiency contributing to vascular abnormalities, and the future research of targeted drugs and therapies to prevent aneurysmal growth and rupture. Given the sex differences in cerebral aneurysms, this review article allows readers to understand risk prediction for individual patients and populations of women with cerebral aneurysms at various stages of their life, from pregnancy to menopause.

\section{References}

1. Barbarite E, Hussain S, Dellarole A, Elhammady MS, Peterson E: The management of intracranial aneurysms during pregnancy: a systematic review. Turk Neurosurg 26:465474, 2016

2. Barrett JM, Van Hooydonk JE, Boehm FH: Pregnancyrelated rupture of arterial aneurysms. Obstet Gynecol Surv 37:557-566, 1982

3. Chen M, Ouyang B, Goldstein-Smith L, Feldman L: Oral contraceptive and hormone replacement therapy in women with cerebral aneurysms. J Neurointerv Surg 3:163-166, 2011

4. de Rooij NK, Linn FH, van der Plas JA, Algra A, Rinkel GJ: Incidence of subarachnoid haemorrhage: a systematic review with emphasis on region, age, gender and time trends. J Neurol Neurosurg Psychiatry 78:1365-1372, 2007

5. Dias MS, Sekhar LN: Intracranial hemorrhage from aneurysms and arteriovenous malformations during pregnancy and the puerperium. Neurosurgery 27:855-866, 1990

6. Ding C, Toll V, Ouyang B, Chen M: Younger age of menopause in women with cerebral aneurysms. J Neurointerv Surg 5:327-331, 2013

7. Hoh BL, Rojas K, Lin L, Fazal HZ, Hourani S, Nowicki $\mathrm{KW}$, et al: Estrogen deficiency promotes cerebral aneurysm rupture by upregulation of Th17 cells and interleukin-17A which downregulates E-cadherin. J Am Heart Assoc 7:7, 2018
8. Jamous MA, Nagahiro S, Kitazato KT, Satomi J, Satoh K: Role of estrogen deficiency in the formation and progression of cerebral aneurysms. Part I: experimental study of the effect of oophorectomy in rats. J Neurosurg 103:1046-1051, 2005

9. Jamous MA, Nagahiro S, Kitazato KT, Tamura T, Kuwayama K, Satoh K: Role of estrogen deficiency in the formation and progression of cerebral aneurysms. Part II: experimental study of the effects of hormone replacement therapy in rats. J Neurosurg 103:1052-1057, 2005

10. Juvela S, Poussa K, Porras M: Factors affecting formation and growth of intracranial aneurysms: a long-term follow-up study. Stroke 32:485-491, 2001

11. Kim YW, Neal D, Hoh BL: Cerebral aneurysms in pregnancy and delivery: pregnancy and delivery do not increase the risk of aneurysm rupture. Neurosurgery 72:143-150, 2013

12. Kittner SJ, Stern BJ, Feeser BR, Hebel R, Nagey DA, Buchholz DW, et al: Pregnancy and the risk of stroke. N Engl J Med 335:768-774, 1996

13. Kizilkilic O, Albayram S, Adaletli I, Kantarci F, Uzma O, Islak C, et al: Endovascular treatment of ruptured intracranial aneurysms during pregnancy: report of three cases. Arch Gynecol Obstet 268:325-328, 2003

14. Liu P, Lv X, Li Y, Lv M. Endovascular management of intracranial aneurysms during pregnancy in three cases and review of the literature. Interv Neuroradiol 21:654-658, 2015

15. Maekawa H, Tada Y, Yagi K, Miyamoto T, Kitazato KT, Korai M, et al: Bazedoxifene, a selective estrogen receptor modulator, reduces cerebral aneurysm rupture in ovariectomized rats. J Neuroinflammation 14:197, 2017

16. Meah VL, Cockcroft JR, Backx K, Shave R, Stöhr EJ: Cardiac output and related haemodynamics during pregnancy: a series of meta-analyses. Heart 102:518-526, 2016

17. Meyers PM, Halbach VV, Malek AM, Phatouros CC, Dowd CF, Lawton MT, et al: Endovascular treatment of cerebral artery aneurysms during pregnancy: report of three cases. AJNR Am J Neuroradiol 21:1306-1311, 2000

18. Miller HJ, Hinkley CM: Berry aneurysms in pregnancy: a 10 year report. South Med J 63:279, 1970

19. Nevo O, Soustiel JF, Thaler I: Cerebral blood flow is increased during controlled ovarian stimulation. Am J Physiol Heart Circ Physiol 293:H3265-H3269, 2007

20. Nisson PL, Meybodi AT, Brasiliense L, Berger GK, Golisch $\mathrm{K}$, Benet A, et al: Cerebral aneurysms differ in patients with hysterectomies. World Neurosurg 120:e400-e407, 2018

21. Piotin M, de Souza Filho CB, Kothimbakam R, Moret J: Endovascular treatment of acutely ruptured intracranial aneurysms in pregnancy. Am J Obstet Gynecol 185:1261-1262, 2001

22. Pool JL: Treatment of intracranial aneurysms during pregnancy. JAMA 192:209-214, 1965

23. Robba C, Bacigaluppi S, Bragazzi NL, Bilotta F, Sekhon MS, Bertuetti R, et al: Aneurysmal subarachnoid hemorrhage in pregnancy-case series, review, and pooled data analysis. World Neurosurg 88:383-398, 2016

24. Roman H, Descargues G, Lopes M, Emery E, Clavier E, Diguet A, et al: Subarachnoid hemorrhage due to cerebral aneurysmal rupture during pregnancy. Acta Obstet Gynecol Scand 83:330-334, 2004

25. Tabuchi S: Relationship between postmenopausal estrogen deficiency and aneurysmal subarachnoid hemorrhage. Behav Neurol 2015:720141, 2015

26. Tada Y, Makino H, Furukawa H, Shimada K, Wada K, Liang EI, et al: Roles of estrogen in the formation of intracranial aneurysms in ovariectomized female mice. Neurosurgery 75:690-695, 2014

27. Tamura T, Jamous MA, Kitazato KT, Yagi K, Tada Y, Uno M, et al: Endothelial damage due to impaired nitric oxide bioavailability triggers cerebral aneurysm formation in female rats. J Hypertens 27:1284-1292, 2009 
28. Tanaka H, Katsuragi S, Tanaka K, Iwanaga N, Yoshimatsu J, Takahashi JC, et al: Impact of pregnancy on the size of small cerebral aneurysm. J Matern Fetal Neonatal Med 30:2759-2762, 2017

29. Task Group on Control of Radiation Dose in Computed Tomography: Managing patient dose in computed tomography. A report of the International Commission on Radiological Protection. Ann ICRP 30:7-45, 2000

30. Tiel Groenestege AT, Rinkel GJ, van der Bom JG, Algra A, Klijn CJ: The risk of aneurysmal subarachnoid hemorrhage during pregnancy, delivery, and the puerperium in the Utrecht population: case-crossover study and standardized incidence ratio estimation. Stroke 40:1148-1151, 2009

31. Tutino VM, Mandelbaum M, Takahashi A, Pope LC, Siddiqui A, Kolega J, et al: Hypertension and estrogen deficiency augment aneurysmal remodeling in the rabbit circle of Willis in response to carotid ligation. Anat Rec (Hoboken) 298:1903-1910, 2015

32. Wiebers DO, Whisnant JP, Huston J III, Meissner I, Brown RD Jr, Piepgras DG, et al: Unruptured intracranial aneurysms: natural history, clinical outcome, and risks of surgical and endovascular treatment. Lancet 362:103-110, 2003

33. Yoshida K, Takahashi JC, Takenobu Y, Suzuki N, Ogawa A, Miyamoto S: Strokes associated with pregnancy and puerperium: a nationwide study by the Japan Stroke Society. Stroke 48:276-282, 2017

\section{Disclosures}

The authors report no conflict of interest concerning the materials or methods used in this study or the findings specified in this paper.

\section{Author Contributions}

Conception and design: Khalessi, Desai, Wali, Santiago-Dieppa. Acquisition of data: Desai, Wali. Analysis and interpretation of data: Desai, Wali, Santiago-Dieppa. Drafting the article: all authors. Critically revising the article: Khalessi, Desai, Wali, Santiago-Dieppa. Reviewed submitted version of manuscript: all authors. Approved the final version of the manuscript on behalf of all authors: Khalessi. Statistical analysis: Desai, Wali. Administrative/technical/material support: Khalessi, Desai, Wali. Study supervision: Khalessi, Desai, Wali.

\section{Correspondence}

Alexander A. Khalessi: University of California, San Diego, CA. akhalessi@ucsd.edu. 